\title{
Derecho penal de la empresa a través del role playing
}

\author{
Mariana N. Solari-Merlo ${ }^{a}$
}

${ }^{a}$ Profesora sustituta interina de la Universidad de Cádiz. Departamente de Derecho internacional público, penal y procesal.mariana.solari@uca.es

\begin{abstract}
The present work proposes the gamification of the subject of Corporate Criminal Law of the Degree in Law of the University of Cádiz by means of the implementation of the role playing method. The serious game, according to a proven scientific literature, brings enormous benefits in the acquisition of knowledge as well as in the development of various skills and abilities in the student body, especially highlighting the promotion of a greater degree of autonomy in the learning process.

In the proposed role play, the students, in a collaborative way, build their own knowledge around a practical assumption. This common base will be on which they must expand their investigations to solve the problems discussed, this time, based on the role assigned to each one. The roles worked, lawyer, prosecutor and judge, reproduce some of the most common professions to which the law student accesses. In this sense, by simulating a trial, each student can experience first-hand the structure and operation of the prosecution of a criminal case while exercising their oratorical and argumentative skills, which are fundamental in Law.
\end{abstract}

Keywords: role playing, active learning, gamification, role play, corporate criminal law.

\section{Resumen}

Se propone en el presente trabajo la gamificación de la asignatura de Derecho penal de la empresa del Grado en Derecho de la Universidad de Cádiz mediante la implementación del método de role playing. El juego serio, según una contrastada literatura científica, aporta enormes beneficios en la adquisición de conocimientos así como en el desarrollo de diversas destrezas y habilidades en el estudiantado, destacando especialmente el fomentar un mayor grado de autonomía en el proceso de aprendizaje.

En el juego de rol propuesto, los alumnos, de modo colaborativo, van construyendo su propio conocimiento en torno a un supuesto práctico. Esta base común será sobre la que deban ampliar sus investigaciones para resolver los problemas tratados, esta vez, en base al rol asignado a cada uno. Los roles trabajados, abogado, fiscal y juez, reproducen algunas de las profesiones más habituales a las que accede el estudiante de Derecho. En este sentido, mediante la simulación de un juicio, cada alumno puede experimentar en primera persona la estructura y funcionamiento del enjuiciamiento de un caso penal a la vez que ejercitar sus habilidades oratorias y argumentativas, de carácter fundamental en Derecho.

Palabras clave: role playing, aprendizaje activo, gamificación, juego de rol, Derecho penal de la empresa. 


\section{Introducción}

El ámbito universitario resulta un entorno especialmente propicio para la implementación de metodologías docentes que implican un compromiso del alumnado con su propio aprendizaje. El grado de madurez adquirido, especialmente en los últimos cursos de Grados del EEES, así como el vertiginoso salto al que pronto se enfrentarán al acceder al mercado laboral, exige a los docentes una especial labor preparatoria, centrada en el desarrollo de las herramientas personales, resolutivas, que luego les serán necesarias en su vida profesional. Como señala Zumaquero Gil (2018), "no es lo mismo que el propósito del profesor sea simplemente transmitir conocimientos al alumno, convirtiéndose en un mero reproductor de conceptos, que enseñarle a aplicar tales conocimientos o promover la participación, el debate y el intercambio entre ellos".

La metodología de aprendizaje activo se inserta dentro de la teoría del aprendizaje del constructivismo y enfatiza la necesidad de construcción de su propio conocimiento por parte del alumnado (Piaget, 1983). El comportamiento activo por parte del estudiante contribuye a que su aprendizaje se vuelva significativo toda vez que no es un mero destinatario pasivo de la información, sino que se lo fuerza a actuar, pensar, interpretar y aplicar lo aprendido. Como señala González (2000), se expone al alumno a situaciones que requieren operaciones intelectuales superiores, tales como analizar, sintetizar, interpretar o evaluar, entre otras.

Este cambio de comportamiento en el rol asignado a profesor y estudiante tiene un impacto relevante en el proceso de aprendizaje (Bonwell y Eison, 1991; Oltra Mestre, et al., 2012), facilitando tanto la adquisición de nuevos conceptos como la consolidación de los mismos. Es decir, se facilita que la información adquirida se traslade, en primer lugar, de la memoria a corto plazo a la memoria a largo plazo para, con posterioridad, incorporarse en los esquemas de pensamiento más profundo (Wadsworth, 1996). En este sentido, consideramos que el diseño de la estrategia a emplear para alcanzar esta meta debe centrarse en dos factores clave. En primer lugar, procurar conectar los nuevos conocimientos con conocimientos ya adquiridos, fomentando la necesidad de emplear información previa a la nueva situación ya que, como señala Ausuble (1968), esta es la base del aprendizaje significativo. En segundo lugar, se debe fomentar la interacción social de los estudiantes, tanto con sus pares como con el profesorado (Johnson, Johnson \& Smith, 1998), dada la enriquecedora multiplicidad de visiones que se puede dar a un mismo fenómeno.

En este sentido, existen diversos recursos o estrategias habitualmente utilizadas para implementar metodologías activas en el aula. Así, el aprendizaje colaborativo, el aprendizaje basado en problemas, el método de casos, la carpeta de aprendizaje, entre otros (Elena, 2010). Se ha optado, no obstante, por introducir un componente lúdico a través del juego de rol ya que, consideramos, este método aúna los objetivos metodológicos anteriormente descritos. Como señala Ruben (1999), se trata de "una metodología que supera las limitaciones de la enseñanza tradicional... el uso de las simulaciones como metodología educativa conlleva una mejora en el proceso de aprendizaje del propio estudiante, facilita la interactividad entre los estudiantes, el trabajo colaborativo y, en definitiva, un aprendizaje más activo" (García Magna et al., 2011). Asimismo, junto a la consecución de objetivos competenciales específicos relacionados con el contenido de la asignatura, se fomenta una actitud positiva hacia la asignatura elevando la motivación del estudiante al afrontar nuevos retos (Contreras-Espinosa, 2016; Espaliú Berdud, 2017).

Así, a través del denominado juego serio se introduce una dinámica estimulante que "hacen más atractiva la interacción del alumno con el proceso de aprendizaje, con el objetivo de que éste consiga adquirir de forma adecuada determinados resultados" (Fernández Solo de Zaldívar, 2015). Como señalan Linder et al. (2001), el juego serio implica un desarrollo de las habilidades cognitivas, expresión emocional, una vinculación social, añadiendo cierta dosis de competitividad como elemento motivacional (de-MiguelMolina et al., 2015; Fernández Solo de Zaldívar, 2015; de-Miguel-Molina et al., 2019). La opción por el 
juego de rol, a su vez, permite introducir al alumnado en un papel concreto en una simulación controlada (García Magna et al., 2011) debiendo desenvolverse en el ejercicio del modo más eficiente posible siempre dentro de los límites de cada personaje. De este modo, se potencia en el alumno la creatividad, el pensamiento crítico, las habilidades analíticas y el desarrollo de las estrategias más adecuadas para alcanzar la victoria (Krain y Lantis 2006; Jiménez y Moncholi, 2009; Gaete-Quezada, 2011; Espaliú Berdud, 2017).

En el ámbito del derecho, existen numerosas experiencias que introducen el juego de rol y los juicios simulados en la enseñanza aproximando al alumnado al ejercicio de la práctica profesional (por todos, Espaliú Berdud, 2011). En el modelo aquí planteado, los roles a asumir por los estudiantes han sido los de abogado, juez y fiscal, centrándonos en el trabajo de búsqueda y desarrollo de los argumentos jurídicos más adecuados para defender la postura asumida, según se desarrollará a continuación.

\section{Objetivos}

Los objetivos principales de esta experiencia han sido dos:

1. La implicación activa del alumnado en su proceso de aprendizaje

2. La aproximación del alumnado al ejercicio de la práctica jurídica

Asimismo, el siguiente cuadro sintetiza los objetivos secundarios relacionados con los anteriores.

Tabla 1. Resultados del análisis factorial

\begin{tabular}{|c|c|}
\hline $\begin{array}{l}\text { Implicación activa del alumnado en su } \\
\text { proceso de aprendizaje }\end{array}$ & $\begin{array}{c}\text { Aproximación del alumnado al ejercicio } \\
\text { de la práctica jurídica }\end{array}$ \\
\hline Manejo de buscadores jurísprudenciales & $\begin{array}{c}\text { Diseño de la estrategia más adecuada en } \\
\text { base a los conocimientos jurídicos y al rol } \\
\text { asignado }\end{array}$ \\
\hline Majeno de fuentes bibliográficas científicas & Capacidad de síntesis \\
\hline $\begin{array}{c}\text { Detección de las propias carencias } \\
\text { formativas }\end{array}$ & Capacidad argumentativa \\
\hline $\begin{array}{l}\text { Elaboración de los pertinentes documentos } \\
\text { jurídicos según el rol asignado }\end{array}$ & $\begin{array}{l}\text { Correcta expresión oral y soltura en el } \\
\text { empleo de conceptos jurídicos }\end{array}$ \\
\hline
\end{tabular}

\section{Desarrollo de la innovación}

\subsection{Contexto de la propuesta}

La presente propuesta fue pensada para la asignatura de Derecho penal de la empresa de primer cuatrimestre de cuarto curso del Grado en Derecho de la Universidad de Cádiz. En este último curso, el alumnado debe optar el itinerario de Derecho público o por el de Derecho Privado y Derecho de la Empresa, cursando diferentes asignaturas en cada caso. Derecho penal de la empresa es una de las asignaturas optativas que se ofertan en el segundo de los itinerarios mencionados, debiendo el estudiante superar dos asignaturas entre las cinco ofertadas (12 cts. EEES de $30 \mathrm{cts}$. EEES ofertados).

El número de alumnos matriculados suele ser reducido. En el curso 2020/2021 se matricularon 15 alumnos, participando todos ellos en la propuesta metodológica, mientras que el curso 2019/2020 fueron 14 alumnos que, de igual modo, participaron en el sistema que a continuación se describirá. 
Cabe mencionar que, en el presente curso, las clases comenzaron a impartirse con normalidad en el mes de octubre de 2020. La situación de pandemia, no obstante, hizo alterar el desarrollo de la docencia debido a la modificación en el nivel de alerta sanitaria por parte de la Junta de Andalucía en el mes de abril de 2020 (BOJA Extraordinario núm. 77, Decreto del Presidente 9/2020, de 8 de noviembre, por el que se establecen medidas en el ámbito de la Comunidad Autónoma de Andalucía en aplicación del Real Decreto 926/2020, de 25 de octubre, por el que se declara el estado de alarma para contener la propagación de infecciones causadas por el SARS-COV-2 y Orden de 8 de noviembre de 2020, por la que se modulan los niveles de alerta 3 y 4 como consecuencia de la situación crítica epidemiológica derivada del COVID-19 en la Comunidad Autónoma de Andalucía) y a la Resolución Rectoral UCA/R199REC/2020, de 9 de noviembre de 2020, por la que se actualizan las medidas preventivas orientadas a la disminución de la movilidad para minimizar la expansión de la COVID-19 por la que se suspendía la impartición de clases presenciales en Grados sustituyéndolas por clases online en el horario habitual.

En la previsión del curso, no obstante, se tuvo en cuenta esta posible eventualidad introduciéndose diversos recursos TICs como complemento de la docencia. En este sentido, el desarrollo de las clases y, en concreto, de la metodología propuesta no se vio imposibilitado por la situación.

\subsection{Ejecución de la propuesta}

La asignatura tiene dos partes, una primera -más breve- con 4 temas de la parte general del Derecho penal económico, donde se explican los fundamentos para aplicar los delitos, y la segunda parte que es la parte especial, donde vemos los delitos concretos agrupados en temas: ej., delitos contra el medio ambiente, relativos a sociedades, contra la hacienda pública, etc.

La explicación de la primera parte del temario, con una duración aproximada de un mes, tiene una estructura más tradicional aunque haciendo especial hincapié en fomentar la autonomía del alumnado y prepararlo para la metodología que se implementará en la siguiente parte. Así, se trabaja especialmente en el manejo de las herramientas necesarias para resolver los casos a través de la explicación de cuestiones metodológicas, búsqueda de jurisprudencia, artículos de investigación, fuentes disponibles, normas de presentación del trabajo, etc. En este caso, prima el trabajo individual dado que es preciso asegurarse que todo el alumnado adquiere las herramientas necesarias para desarrollar la segunda parte relativa al aprendizaje activo y donde se desarrollará el role playing.

A partir del mes de noviembre se comienza a implementar la presente metodología. Así, en primer lugar, se pone a disposición del alumnado el contenido teórico básico de cada tema que le servirá como punto de partida para la resolución de los supuestos. Cercano a la técnica de flipped classroom, el estudio del material se realiza fuera de clase dejando los primeros minutos de cada sesión para la resolución de dudas por parte del profesorado o la explicación de conceptos que pudieran resultar más complejos.

Finalizada esta parte, se presenta el caso propuesto de la sesión. Se trata de un supuesto jurídico relativo a una situación real concreta relacionada con el tema que corresponde a la sesión. Todo el alumnado accede al supuesto por primea vez en clase y plantea las dudas que pudiera tener respecto a la comprensión del propio caso.

Resuelto esto, se designa los roles con los que los alumnos trabajarán en el caso: abogado, fiscal o juez. La designación de roles es aleatoria para cuya generación se utiliza una web (random.com) que permite generar listas al azar. Conocido su rol, cada alumno deberá analizar el caso, ahora a la luz de la postura asignada buscando los argumentos jurídicos que le sean más favorables. En este punto, las clases se vuelven más dinámicas dado que cada estudiante se puede juntar con sus "colegas" de profesión para debatir el caso y planear, si se estima oportuno, una estrategia común. Cabe mencionar que los alumnos no están obligados 
a compartir la misma postura que sus colegas dado que no se trata de un trabajo en equipo. Aún así, esto suele ser lo habitual.

En este punto, el rol del profesor es asesorar y guiar a los diferentes grupos aproximándose a su punto de reunión -cada grupo se distancia físicamente en el aula de los otros roles. Asimismo, se les cuestiona sobre la estrategia a emplear a la vez que, de modo indirecto, se puede ir comprobando el grado de familiarización de cada estudiante con el tema, dado su reducido número. En el curso 2020/2021, no obstante, con la situación de docencia online comentada, la reunión de expertos se llevó a cabo a través de chats habilitados en el campus virtual de la asignatura donde únicamente tenían acceso quienes compartían el mismo rol y la profesora.

Durante el tiempo restante de clase, los alumnos trabajan en sus grupos buscando información complementaria para resolver la cuestión planteada, fundamentalmente relativa a artículos científicos y, de modo especial, jurisprudencia aplicable.

Hacia la finalización de la clase se procede a la exposición de las posturas de cada rol. Para esto, de modo voluntario en un primer momento, un representante de cada rol sintetiza los principales argumentos jurídicos aplicables respetando el orden establecido en el foro: en primer lugar, habla la acusación, a continuación la defensa y, finalmente, el juez da su veredicto. Cabe destacar que, al no tratarse de un trabajo en grupo como se ha señalado, el alumno que mantenga una opinión discordante respecto a sus colegas, debe pedir la palabra para intervenir una vez finalice la exposición del representante del rol. Dada la relevancia de la argumentación jurídica y de las habilidades oratorias en Derecho, esta fase resulta especialmente relevante para que los alumnos puedan ejercitar dichas competencias en una asignatura que, cabe recordar, se imparte en el último año de su formación. Asimismo, el número reducido de alumnos con los que cuenta la asignatura resulta especialmente propicio para aquellos alumnos con más dificultades a la hora de expresarse en público o en el manejo del vocabulario jurídico. En este sentido, el profesorado procura que el expositor representante de cada rol rote semanalmente.

Al finalizar las exposiciones, el profesorado resuelve las dudas que pudieran persistir sobre la aplicación material del Derecho y comenta los argumentos jurídicos más favorables a cada parte, hayan reparado en esto los estudiantes o no.

Dejando un día de gracia, cada alumno debe subir al campus virtual el pertinente documento jurídico que, de alguna forma, viene a certificar su trabajo. Según el rol asignado, se realizará de modo individual un escrito de acusación, un escrito de defensa o una sentencia.

A la semana siguiente, mantenido las agrupaciones de alumnos, se alternan los roles. Cuando transcurren tres sesiones $-\mathrm{y}$ todos los alumnos pasaron por los tres roles- se vuelve a utilizar la web random.com para generar nuevas agrupaciones a efectos de evitar que el alumno trabaje con los mismos compañeros durante todo el curso. Con esto se pretende fomentar la adaptación de los estudiantes a nuevos entornos y su capacidad de trabajo en equipo, especialmente difícil cuando la elección de compañeros depende de terceros, tal y como sucederá cuando accedan al mundo laboral.

\section{Resultados y propuestas de mejora}

Los resultados en estos dos cursos de impartición resultan altamente satisfactorios, superando la asignatura el 100\% de alumnos que siguió la metodología en el curso 2019/2020 -un estudiante prefirió no hacerlo- y un $93,3 \%$ en el curso 2020/2021 -todos los alumnos siguieron el método pero uno no superó. En este sentido, es de destacar la elevada implicación de los estudiantes en la preparación de los casos y las destrezas adquiridas al finalizar el curso, tal y como han destacado ellos mismos.

(cc) BY-NC-ND 2021, Universitat Politècnica de València

Congreso In-Red (2021) 
No obstante, en base a esta experiencia y, en cierto sentido, a lo aprendido durante el tiempo de docencia online, consideramos que se pueden introducir algunas mejoras de cara a facilitar la colaboración entre alumnos, habituarlos en el manejo -con soltura- de las TICs y, en cierto sentido, hacer más real la experiencia.

De modo resumido, cabe destacar:

- Mayor trabajo colaborativo. Los trabajos no serán individuales como hasta ahora sino que los roles asignados conformarán equipos. Esto no debe opacar el esfuerzo individual ni la necesidad de reprimir la discrepancia con la mayoría. En el escrito final que presenten, el alumno que no esté de acuerdo con sus compañeros debe manifestarlo mediante un voto particular, tal y como se hace en el foro.

- Mayor tiempo para la reflexión y análisis. Los supuestos no se resolverán en la misma sesión sino que se introducirán plazos para que los alumnos suban, escalonadamente, sus escritos el campus virtual. Dado que el trabajo será grupal, se subirá un escrito por rol debiendo constar, en su caso, las opiniones discrepantes de la mayoritaria, como se ha mencionado.

- Lograr mayor interacción entre los roles. Al presentar sus trabajos por turnos, el juego se acercará más a la realidad dado que, en primer lugar subirá su escrito la acusación debiendo la defensa responder expresamente a sus argumentos. Hasta ahora esto no era posible dado que, al resolverse en la misma sesión, los alumnos se centran en su propia investigación sin tener tiempo material para evaluar y responder a las acusaciones. Del mismo modo, los jueces -últimos en subir sus escritos- incorporarán los argumentos esgrimidos tanto por la acusación como por la defensa, analizándolos y determinando su adecuación al supuesto.

- Mantener la motivación. Mientras que a lo largo de la semana los fiscales y jueces subirán sus escritos al foro de la asignatura, para que sean visibles para todos los compañeros, los jueces no darán su veredicto sino hasta la próxima sesión. Así, en la semana siguiente a la presentación del caso, se simulará el juicio con la intervención de las partes que no saben qué decisión adoptará el tribunal, aportando cierta dosis de motivación al tener que esforzarse por argumentar de modo convincente ante este.

- Asimismo, cada grupo deberá utilizar la plataforma padlet (o similar) como apoyo educativo a la hora de realizar anotaciones destacadas o planear su estrategia. Se fomentará también el uso de otras plataformas tipo bubble.es -que ofrece la posibilidad de realizar cuadros sinópticos con un componente visual que aporta claridad- para aclarar las ideas principales de cada tema. Estos recursos se utilizarán de modo colaborativo dentro de cada grupo, permitiendo el acceso al profesorado para, en su caso, comprobar el progreso.

\section{Conclusiones}

La presente propuesta está dirigida a aproximar al alumno en una experiencia simulada de lo que será su próxima experiencia en el ejercicio profesional. A través de un componente lúdico de role playing, el estudiantado puede no sólo conocer los contenidos materiales, curriculares, de la asignatura sino también habituarse a los requerimientos de las principales salidas profesionales del estudiante en Derecho. Mediante estas simulaciones, el alumno puede anticipar el conocimiento de una realidad a la cual, en algunos casos, se aproximará mediante la realización de la asignatura de prácticum; en otros tendrá acceso únicamente una vez finalizados sus estudios.

Es de destacar especialmente el trabajo con las habilidades oratorias y argumentativas de los estudiantes, dada la máxima relevancia que estas destrezas tienen para los juristas. Así, con independencia de la 
profesión por la que se opte, el profesional del Derecho debe ser capaz de transmitir de un modo claro y preciso sus argumentos ante cualquier audiencia, por lo que su trabajo con alumnos de último curso resulta especialmente relevante.

\section{Referencias}

Ausuble, D. P. (1968). Educational psycology: a cognitive view. New York: Holt, Rinehart \& Winston.

Bonwell, C.; Eison, J. Active Learning: Creating Excitement in the Classroom, (1991). AEHEERIC Higher Education Report, núm. 1.

Contreras-Espinosa, R. S. "Presentación. Juegos digitales y gamificación aplicados en el ámbito de la educación" (2016). RIED. Revista Iberoamericana de Educación a Distancia, núm. 19 (2), pp. 27-33.

de-Miguel-Molina et al. Teaching Open Innovation based on LSP: a practical experience (2015). 1st International Conference on Business Management Project: Teaching Management and related subjects.

de-Miguel-Molina et al. Aprendiendo liderazgo y trabajo en equipo mediante el juego fuera del aula (2019). Congreso In-Red 2019 UPV.

Elena García, P. El aprendizaje activo en traducción y su evaluación (2011). Estudios de traducción, núm. 1, pp. 171-183.

Espaliú Berdud, C. La aplicación del juego de roles a la enseñanza del derecho de la Unión Europea (2017). Revista de educación y derecho. Education and law review, núm. 15.

Fernández Solo de Zaldívar, I. Juego serio: gamificación y aprendizaje (2015). Comunicación y Pedagogía: nuevas tecnologías y recursos didácticos, núm. 281-282, (Ejemplar dedicado a: Gamificación). < http://www.centrocp.com/juego-serio-gamificacion-aprendizaje/ > [Consulta: 26 de marzo de 2021]

Gaete-Quezada, R. A. El juego de roles como estrategia de evaluación de aprendizajes universitarios (2011). Educ.Educ., vol. 14, núm. 2, pp. 289-307.

García Magna, D. et al. La interdisciplinariedad en la educación superior: propuesta de una guía para el diseño de juegos de rol (2011). Teoría de la Educación,12(1)27.

González Zamora, H. (2000). La evaluación de los estudiantes en un proceso de aprendizaje activo. Colombia: Centro de recursos para el aprendizaje.

Jiménez Martín, S. y Moncholi Chaparro, M.A. El entrenamiento en técnicas creativas en el Espacio Europeo de Educación Superior (2009). Actas del I Congreso Internacional Latina de Comunicación Social (pp. 1-12). Tenerife: Universidad de La Laguna.

Linder, M.O., Roos, J. y Victor, B. Play in organizations (2001). Working Paper 2. Imagination Lab.

Oltra Mestre, M. J. et al. Aprendizaje activo y desempeño del estudiante: diseño de un curso de dirección de la producción (2012). Working Papers on Operations Management, vol 3, núm. 2, pp. 84-102.

Wadsworth, B. J. (1996). Piaget's Theory of Cognitive and Affective Development. Nueva York: Longman.

Zumaquero Gil, L. Los juegos de rol como estrategia de enseñanza-aprendizaje para fomentar la adquisición de competencias: una experiencia en la titulación de grado en turismo (2018). REJIE Nueva época: Revista Jurídica de Investigación e Innovación Educativa, núm.18, Junio, pp. 43-56. 